\title{
Simulation of continuum heat conduction using DEM domains
}

\author{
I. TERREROS ${ }^{\mathrm{a}}$, I. IORDANOFF ${ }^{\mathrm{a}, \mathrm{b}}$, J. L. CHARLES ${ }^{\mathrm{b}}$ \\ ${ }^{a}$ Arts et Métiers ParisTech, I2M-MPI, UMR 5295 CNRS, France \\ ${ }^{b}$ Arts et Métiers ParisTech, I2M-DuMAS, UMR 5295 CNRS, France
}

\begin{abstract}
Currently, almost all material manufacturing processes are simulated using methods based on continuum approaches such as the Finite Element Method (FEM). These methods, though widely studied, face difficulties with multibody, contact, high-strain and high-displacement problems, which are usually found in manufacturing processes. In some cases, the Discrete Element Method (DEM) is used to overcome these problems, but it is not yet able to simulate some of the physics of a continuum material, such as 3D heat transfer.

To carry out a realistic simulation of a process, its thermal field must be properly predicted. This work describes a fast and efficient method to simulate heat conduction through a 3D continuum material using the Discrete Element Method. The material is modelled with spherical discrete elements of different sizes to obtain a compact and isotropic domain adequate for carrying out mechanical simulations to obtain straightforward thermal and mechanical coupling.

Thermal simulations carried out with the proposed Discrete Element Method are compared to both the analytical and FEM results. This comparison shows excellent agreement and validates the proposed method.
\end{abstract}

Keywords:

Discrete Element Method, Heat conduction, Thermal field, Transient temperature, Continuum materials.

\section{Introduction}

The importance of temperature fields in almost any manufacturing method is indisputable. Due to the very strong dependence of metallurgical phenomena on these fields, they critically influence the mechanical properties of the processed material. Consequently, the search for a realistic thermal simulation of a process, including accurate temperature predictions, is an issue of capital importance for the desired full control of manufacturing techniques.

A law for heat conduction through a continuous material equation 1 in the case of constant conductivity where $\Theta$ is the temperature, $\lambda$ is the thermal conductivity, $\rho$ is the density of the material, and $t$ represents the time - was first proposed by FourIER in 1822 [1]. This equation provides analytical solutions for some geometrically simple problems [2] and has been widely solved using various numerical methods, but primarily by the Finite Element Method [3] due to its natural adaptation to partial differential equations.

$$
\frac{\partial \Theta}{\partial t}=\frac{\lambda}{\rho c_{p}}\left(\frac{\partial^{2} \Theta}{\partial x^{2}}+\frac{\partial^{2} \Theta}{\partial y^{2}}+\frac{\partial^{2} \Theta}{\partial z^{2}}\right)
$$

In tooling processes, regions with high thermal gradients and heat fluxes are often located near the tool-piece contact. In the contact area, continuous approaches have difficulty accurately

Email addresses: inigo.terreros@u-bordeaux1.fr(I. TERREROS), ivan. iordanoff@ensam.eu (I. IORDANOFF),

j1. charles@i2m.u-bordeaux1.fr (J. L. CHARLES) describing high strains and temperature gradients. In this area, the Discrete Element Method [4] is a good alternative to the FEM to locally solve problems related to material fracture [5], multibody system coupling [6] or dry sliding contact with a third body presence [7], among others. A long term solution consists of solving the contact area with the DEM and solving the piece and tool far from the contact with continuous approaches, such as the FEM or the NEM [8], and then coupling these methods (figure 1).

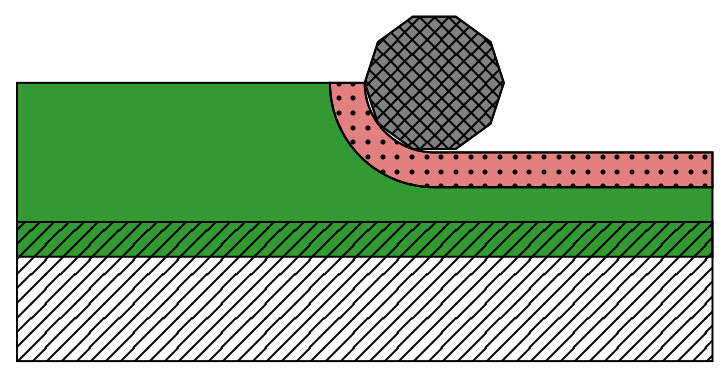

Figure 1: Simplified example of a milling process. Discrete Element Method is used in the zone in contact with the tool (dotted) in order to simulate complex thermo-mechanical behaviours. DEM is also used beyond the contact zone (solid) in order to ease the coupling with a Finite Element Method zone (striped).

There are many works that describe the use of the Discrete Element Method in a mechanical field such as [9], which uses LENNARD-JONES potentials [10] to describe the interaction forces between discrete elements, the work in reference [11], where discrete elements are linked in a truss, and exact results for the 
stress and strain fields of 2D shells are obtained, or [12], which links discrete elements by beams and describes how to carry out wear simulations for brittle elastic materials.

However, examples of the DEM in thermal fields are mostly focused on problems involving granular materials [7, 13, 14, $15,16,17]$, with the exception of [18], in which the mathematical proof of the 2D DEM for a continuous thermal field is described.

The present work describes a method to simulate isotropic heat conduction through a $3 \mathrm{D}$ continuum material using the Discrete Element Method. The material is modelled with spherical discrete elements of different sizes to obtain a compact and isotropic domain adequate for carrying out 3D mechanical simulations [12]. The validity of the described method in such a discrete domain is the basis for thermo-mechanical simulations using the DEM.

The paper is structured as follows: In Section 2 a description of the method is given. This method is proved in Section 3 for $3 \mathrm{D}$ crystal domains and in Section 4 for isotropic domains. In Section 5 numerical results are shown and discussed. Finally, conclusions are inferred in Section 6.

\section{General description of the method}

Figure 2 shows a typical discrete domain created exclusively with spherical discrete elements.
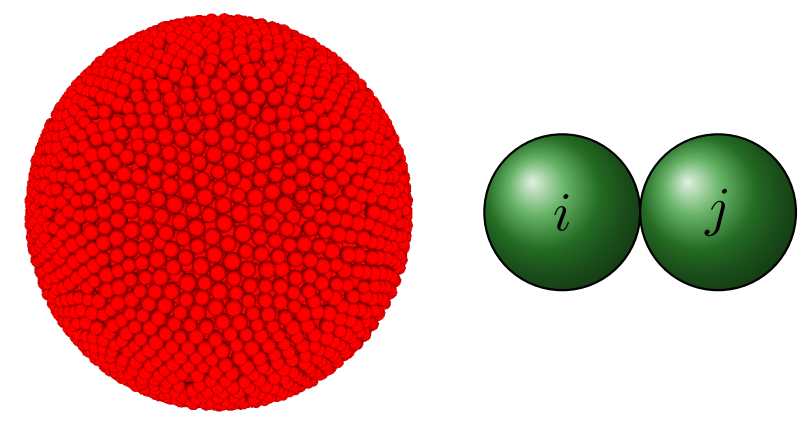

Figure 2: A sample of a spherical discrete domain and an example of neighbour discrete elements.

To find the variation of a given magnitude through a discrete domain, there are two principal steps to follow, as explained in [13]:

- First, variations due to the interaction between each discrete element and all its neighbours must be separately analysed and stored.

- Then, stored variations of each discrete element must be summed to obtain the total variation of the magnitude for the discrete element.

Mathematically:

$$
G_{i}=\sum^{N_{\text {neigh }}} g_{i j}
$$

where $G_{i}$ is the variation of the measured magnitude for the discrete element $i$ and $g_{i j}$ is the variation of the magnitude of discrete element $i$ due to its interaction with its neighbour $j$.

\subsection{Application to heat conduction}

In the particular case of heat conduction, transferred heat between two discrete elements for a given instant, $W_{i j}$, can be calculated using Fourier's law as follows:

$$
W_{i j}=S_{t} \lambda \frac{\left(\theta_{j}-\theta_{i}\right)}{d_{i j}}
$$

where $S_{t}$ is the heat transmission surface's area; $\left(\theta_{j}-\theta_{j}\right)$ is the temperature difference between discrete element $i$ and discrete element $j ; \lambda$ is the material's heat conductivity and $d_{i j}$ is the distance between discrete element $i$ and discrete element $j$.

Furthermore, the thermal energy gain of a discrete element $i$, $\Delta E_{i}$ in the equation, can be calculated as a function of its rise in temperature:

$$
\Delta E_{i}=c_{p} \rho_{d} V_{i} \Delta \theta_{i}
$$

where $c_{p}$ is the specific thermal capacity of the discrete element; $\rho_{d}$ represents the discrete element's density; $V_{i}$ is the volume and $\Delta \theta_{i}$ is the raise of temperature of a discrete element $i$.

The time derivative of the thermal energy gain represents the heat being transferred to discrete element $i$ :

$$
\frac{\Delta E_{i}}{\Delta t}=W_{i j}=\frac{c_{p} \rho_{d} V_{i} \Delta \theta_{i}}{\Delta t}
$$

If the time step $\Delta t$ is small enough to consider $\left(\theta_{j}-\theta_{i}\right)$ constant during the time step, equations 3 and 4 can be combined to obtain the temperature rise of discrete element $i$ due to its interaction with a neighbour $j$ :

$$
\Delta \theta_{i}=\frac{\left(\theta_{j}-\theta_{i}\right) S_{t} \lambda}{d_{i j} c_{p} \rho_{d} V_{i}} \Delta t
$$

Finally, equation 2 is used to obtain $\Delta \Theta_{i}$, the total variation of the temperature of discrete element $i$, after the time step $\Delta t$ :

$$
\Delta \Theta_{i}=\sum^{N_{\text {neigh }}} \Delta \theta_{i}
$$

\section{Proof of concept on 3D crystal domains}

In the work by Hahn et al. [18], a method to predict temperature fields in 2D discrete domains formed by hexagonal discrete elements is proved. In this section a proof for $3 \mathrm{D}$ discrete domains formed by identically-sized spherical discrete elements placed following a simple cubic crystal pattern will be given, following the method described in paragraph 2.1.

\subsection{Proof}

Figure 3 represents an example of the domain described above. To fill all of the domain's volume, each discrete element represents a cube of continuous material whose volume equals $(2 R)^{3}$.

Equation 5 can be modified for the particular case of the crystal domain of figure 3 as follows:

First, the mass of each discrete element must equal the mass of the represented volume. Then, the discrete element's density 


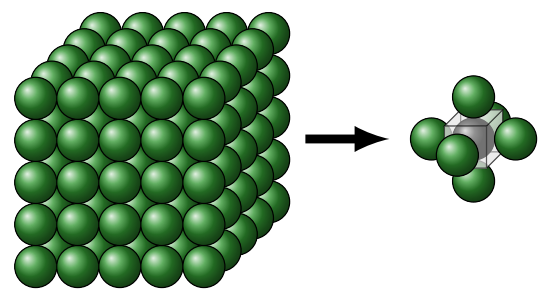

Figure 3: 3D crystal domain and example of a random particle with six neighbours. The volume represented by each discrete element is $2 R \times 2 R \times 2 R=8 R^{3}$.

$\rho_{d}$ is linked to the material's density $\rho_{c}$ by means of the volume fraction $f_{v}$ following:

$$
\rho_{d}=\frac{\rho_{c}}{f_{v}}=\frac{V_{\text {cube }}}{V_{\text {sph }}} \rho_{c}=\frac{8 R^{3}}{4 / 3 \pi R^{3}} \rho_{c}=\frac{6}{\pi} \rho_{c}
$$

Let us consider the transmission surface's area to be equal to the surface of the cube face $S_{t}=4 R^{2}$ and the distance between discrete elements equal to the cube side $d_{i j}=2 R$.

Equation 5 then becomes:

$$
\begin{aligned}
\Delta \theta_{i} & =\frac{\left(\theta_{j}-\theta_{i}\right) S_{t} \lambda}{d_{i j} c_{p} \rho_{d} V_{s p h}} \Delta t=\frac{\left(\theta_{j}-\theta_{i}\right)\left(4 R^{2}\right) \lambda}{(2 R) c_{p} \rho_{c}\left(V_{\text {cube }} / V_{s p h}\right) V_{s p h}} \Delta t \\
& =\frac{\lambda}{c_{p} \rho_{c}} \frac{\left(\theta_{j}-\theta_{i}\right)}{(2 R)^{2}} \Delta t
\end{aligned}
$$

Let us consider the six discrete elements around discrete element $i$ (figure 4). The total temperature variation is then given by:

$$
\Delta \Theta_{i}=\sum^{6} \Delta \theta_{i}=\frac{\lambda}{\rho_{c} c_{p}} \frac{\left(\theta_{1}+\theta_{2}+\theta_{3}+\theta_{4}+\theta_{5}+\theta_{6}-6 \theta_{i}\right)}{(2 R)^{2}} \Delta t
$$

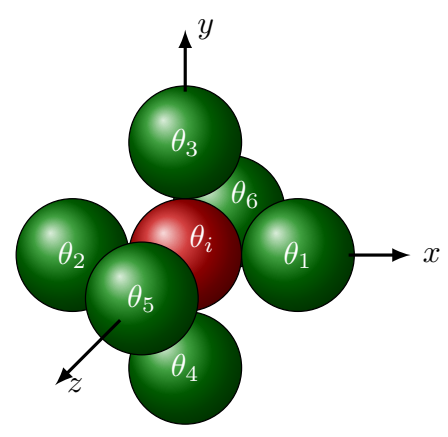

Figure 4: Six discrete elements around a discrete element $i$.

Therefore:

$$
\begin{aligned}
\Delta \Theta_{i} & =\sum^{6} \Delta \theta_{i} \\
& =\frac{\lambda}{\rho_{c} c_{p}}\left(\frac{\theta_{1}+\theta_{2}-2 \theta_{i}}{(2 R)^{2}}+\frac{\theta_{3}+\theta_{4}-2 \theta_{i}}{(2 R)^{2}}+\frac{\theta_{5}+\theta_{6}-2 \theta_{i}}{(2 R)^{2}}\right) \Delta t
\end{aligned}
$$

Which is an exact fit to the finite central difference stencil for the $3 \mathrm{D}$ heat equation (equation 1 ) with a mesh size equal to $2 R$ :

$$
\begin{aligned}
\frac{\partial \Theta}{\partial t} & =\frac{\lambda}{\rho_{c} c_{p}}\left(\frac{\partial^{2} \Theta}{\partial x^{2}}+\frac{\partial^{2} \Theta}{\partial y^{2}}+\frac{\partial^{2} \Theta}{\partial z^{2}}\right) \approx \\
& \approx \frac{\lambda}{\rho_{c} c_{p}}\left(\frac{\theta_{1}+\theta_{2}-2 \theta_{i}}{(2 R)^{2}}+\frac{\theta_{3}+\theta_{4}-2 \theta_{i}}{(2 R)^{2}}+\frac{\theta_{5}+\theta_{6}-2 \theta_{i}}{(2 R)^{2}}\right)
\end{aligned}
$$

\subsection{Conclusions}

The use of equation 2 to describe heat conduction through a $3 \mathrm{D}$ continuous material modelled with a discrete crystalline domain fits a central difference stencil if:

- The mass of each discrete element must be equal to the mass of the discretized continuous material.

- The transmission surfaces are considered to be equal to the surfaces of the polyhedron surrounding each discrete element (a cube in the given example).

\section{Extension to 3D isotropic domains}

To ease the coupling of the thermal and mechanical aspects of a simulation, both the thermal DEM and mechanical DEM should work together using the same discrete domain. To assure the quantitative simulation of a solid material by the use of the DEM, the work in reference [12] shows that the discrete domain must be compact, homogeneous and isotropic (in terms of the contact directions). For this purpose, a size repartition greater than $15 \%$ around the main diameter must be chosen [12, 19]. The radii used in this work are generated using an uniform probability density between $R_{m}(1-0.15 / 2)$ and $R_{m}(1+0.15 / 2)$.

To extend the results of paragraph 2.1 to isotropic domains, the conclusions inferred in section 3.2 will be supposed to be necessary, even if the structure of the $3 \mathrm{D}$ discrete domain differs.

The problem is that there is no geometrical method for finding transmission surfaces and volume fractions (figure 5). So, parameters $S_{t}$ and $\rho_{d}$ from equation 5 must be locally obtained using a robust and numerically efficient method that constitutes the main originality of the present work. The use of this 3D method is in agreement with $2 \mathrm{D}$ results of [18] that show that "local anisotropy in the thermal heat conductivity on a small scale has no effect on the heat flux on the macroscale".

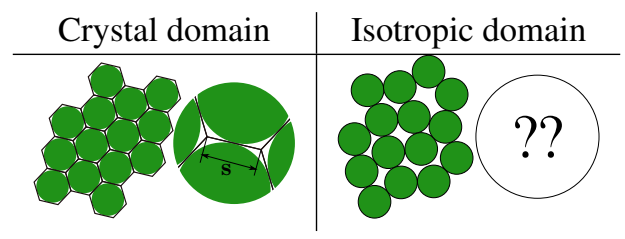

Figure 5: 2D example of a crystal domain - known transmission surface \& known volume fraction - and an isotropic domain - unknown transmission surface \& unknown volume fraction. 


\subsection{Determination of local parameters for each discrete ele- ment}

To determine the transmission surface's area and the discrete element's density in an isotropic domain, an equivalent platonic solid - that depends on the number of neighbours - is considered for each discrete element. An example is shown in figure 6.

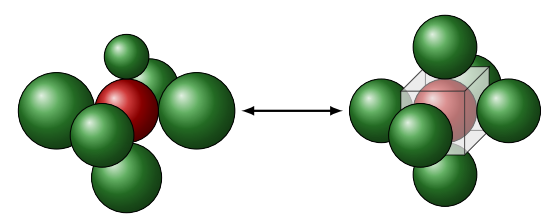

Figure 6: Example of an equivalent regular polyhedron.

The transmission surfaces areas and local volume fractions are calculated from this platonic solid. (Due to the random packing of discrete elements, the volume fraction may vary from one discrete element to another, consequently, the concept of "local" volume fraction is now used instead of the concept of volume fraction used before.)

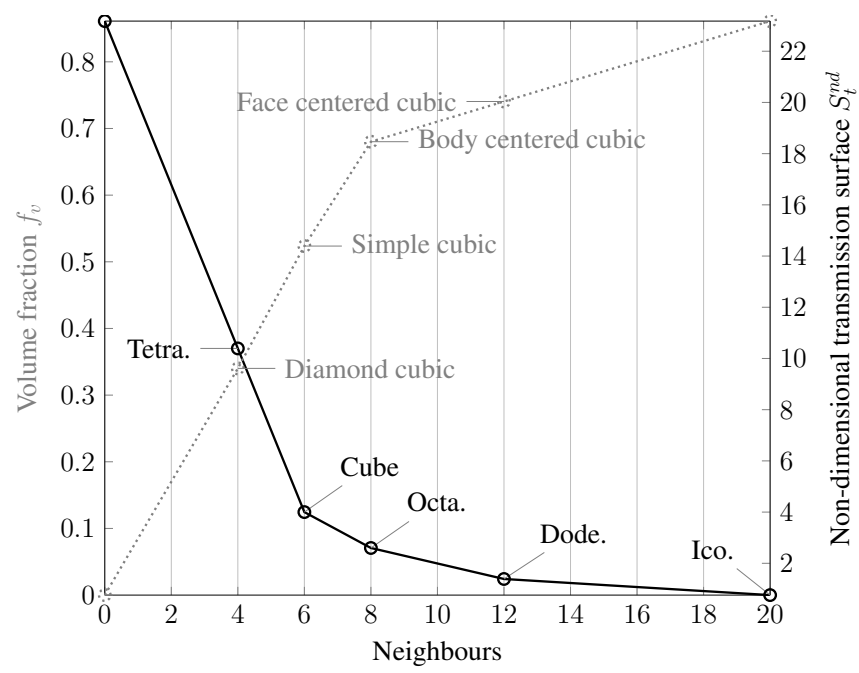

Figure 7: Non-dimensional transmission surfaces (solid curve) and volume fractions (dotted curve) as a function of the number of neighbours.

\begin{tabular}{rllrll}
\hline$N_{\text {neigh }}$ & $S_{t}^{\text {nd }}$ & $f_{v i}$ & $N_{\text {neigh }}$ & $S_{t}^{\text {nd }}$ & $f_{v i}$ \\
\hline 1 & 19.98076 & 0.08502 & 11 & 1.69019 & 0.72540 \\
2 & 16.78460 & 0.17004 & 12 & 1.38757 & 0.74048 \\
3 & 13.58845 & 0.25506 & 13 & 1.30888 & 0.75555 \\
4 & 10.39230 & 0.34008 & 14 & 1.23020 & 0.77063 \\
5 & 7.19615 & 0.43184 & 15 & 1.15152 & 0.78570 \\
6 & 4.0 & 0.52359 & 16 & 1.07284 & 0.80078 \\
7 & 3.29903 & 0.60188 & 17 & 0.99415 & 0.81586 \\
8 & 2.59807 & 0.68017 & 18 & 0.91547 & 0.83093 \\
9 & 2.29545 & 0.69525 & 19 & 0.83679 & 0.84601 \\
10 & 1.99282 & 0.71032 & 20 & 0.75810 & 0.86109 \\
\hline
\end{tabular}

Table 1: Calculated values for $S_{t}^{n d}$ and $f_{v}$ as a function of the number of neighbours.

\subsection{Calculation of discrete element's transmission surface}

As stated in paragraph 4.1, discrete element $i$ is related to a regular polyhedron (platonic solid) by means of its number of neighbours. The number of surfaces of the chosen polyhedron is equal to this number. Then, the area of that polyhedron's face is calculated and used as the transmission surface area of discrete element $i$. In the cases where such a regular polyhedron does not exist, the transmission surface is chosen by linear interpolation (figure 7). For the cases where the discrete element is in contact with less than four neighbours, the slope is considered constant. In the example of figure 6 , in which six discrete elements are in contact, the transmission surface's area is considered to be four times the square of its radius $(2 R)^{2}$, as shown in figure 7 and in table 1 .

\subsection{Calculation of local volume fraction}

A similar method is used to calculate the volume fraction occupied by the discrete element. In this case, the number of neighbours is related to the kissing number of known crystal structures. Once a crystal structure is "linked" to the discrete element by means of its number of neighbours, the volume fraction of that crystal structure is used as the discrete element's volume fraction to carry out future calculations. Just as in paragraph 4.2, if the number of neighbours does not match any crystal structure, the local volume fraction is calculated using a linear interpolation (figure 7). For the theoretical case where the discrete element has no neighbours, the local volume fraction is considered to be 0 . Furthermore, when the discrete element of reference is in contact with more than 12 neighbours, the slope is considered constant. For instance, the discrete element shown in figure 6 will occupy nearly $52.359 \%$ of the space, as will an atom in a simple cubic unit cell (figure 8). Figure 7 and table 1 gives the results for any other neighbourhood configuration.

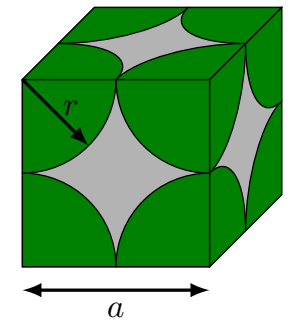

Figure 8: Example of a simple cubic unit cell. $f_{v}=\frac{a^{3}}{(4 / 3) \pi r^{3}}=\frac{(2 r)^{3}}{(4 / 3) \pi r^{3}}=\frac{6}{\pi} \approx$ 0.52359 .

The methods described in paragraphs 4.2 and 4.3 are numerically very efficient. The knowledge of the number of neighbours directly provides the transmission surface and local volume fraction that must be employed for the heat transfer calculation.

\subsection{Interactions between each discrete element and its neigh- bours}

Once the transmission surfaces and local volume fractions are obtained for each discrete element, some considerations must be taken into account to calculate the temperature rise. 
First, the heat exchange between any discrete element $i$ and its neighbour $j$ must be the same, irrespective of the identity of the discrete element of reference. This requirement means that the resultant amount of exchanged heat (equation 3) must be the same, whether reference element $i$ or element $j$ is used in the calculation. This equivalence is achieved by averaging the parameter $S_{t}$ from equation 3 . On the contrary, the parameter $f_{v}$ must not be averaged in such a way, because it determines the temperature rise of the discrete element and this variation depends on the properties of the discrete element itself and not on its interactions.

The transmission surface's area is calculated for discrete elements $i$ and $j$ using the non-dimensional values taken from table 1 ( $S_{i}=S_{i}^{n d} R_{i}^{2}$, for example). Then, the equivalent transmission surface area between the two discrete elements is calculated by means of a geometric average $S_{i j}=\sqrt{S_{i} S_{j}}$.

Taking into account the results of this section, equation 5 can be expressed as follows if the unknown $\rho_{d}$ is substituted by the now known $\rho_{c} / f_{v i}$ (equation 6 , where $f_{v i}$ is the volume fraction, $f_{v}$, of the discrete element $i$ ):

$$
\Delta \theta_{i}=\frac{\left(\theta_{j}-\theta_{i}\right) S_{i j} \lambda f_{v i}}{d_{i j} \rho_{c} c_{p} V_{i}} \Delta t
$$

and the temperature variation after a time step $\Delta t$ of a discrete element $i$ belonging to an isotropic domain can be then described as:

$$
\Delta \Theta_{i}=\sum_{j=0}^{N_{\text {neigh }}} \Delta \theta_{i}=\sum_{j=0}^{N_{\text {neigh }}} \frac{\left(\theta_{j}-\theta_{i}\right) S_{i j} \lambda f_{v i}}{d_{i j} \rho_{c} c_{p} V_{i}} \Delta t
$$

\section{Validation}

To validate the method described in the previous section, two different types of simulations were carried out.

\subsection{Cylindrical beam in contact with a hot plane}

In the first case, the cylindrical domain shown in figure 9, with an initial temperature of $298 \mathrm{~K}$, is submitted to a boundary temperature of $798 \mathrm{~K}$ at $x=0$. There is no heat exchange trough the rest of the surfaces. Further characteristics of the beam are given in table 2 .

\begin{tabular}{ll}
\hline Parameter & Value \\
\hline Length & $2.184 \mathrm{~mm}$ \\
Radius & $0.116 \mathrm{~mm}$ \\
Initial temperature & $298 \mathrm{~K}$ \\
Density & $2790 \mathrm{~kg} / \mathrm{m}^{3}$ \\
Specific heat & $880 \mathrm{~J} /(\mathrm{kg} \mathrm{K})$ \\
Thermal conductivity & $134 \mathrm{~W} /(\mathrm{m} \mathrm{K})$ \\
Discrete elements & 19465 \\
Elements' average radius & $8.998 \mu \mathrm{m}$ \\
\hline
\end{tabular}

Table 2: Beam's characteristics

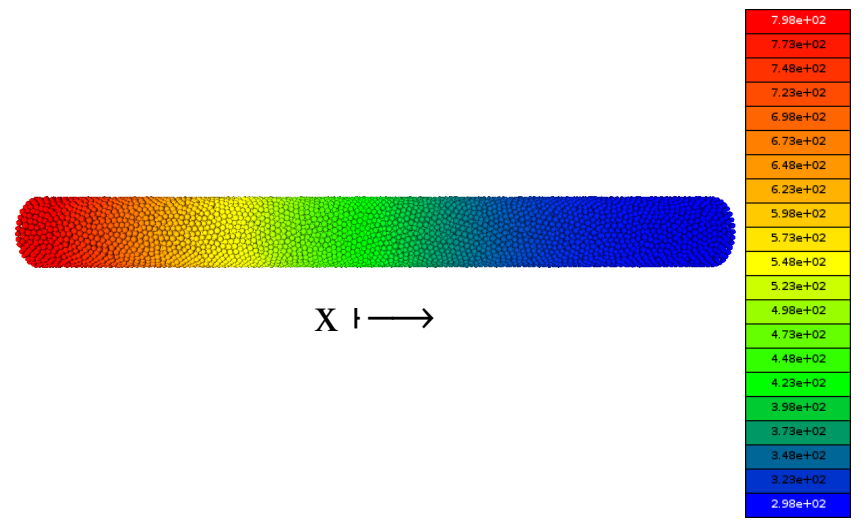

Figure 9: Beam used in the calculations at a given time of the simulation. Color scale represents temperature from $298 \mathrm{~K}$ (in blue) to $798 \mathrm{~K}$ (in red).

The results obtained with the DEM simulation are compared to analytical results from [2] (equation 8, where $\Theta_{M}$ is $798 \mathrm{~K}$ and $\Theta_{m}$ is $298 \mathrm{~K}$ in our particular case) and plotted in figure 10. The simulation and its analytical solution are in good agreement, both at the beginning of the simulation when the greatest temperature gradient is found and at the end of the simulation when the temperature gradient is least.

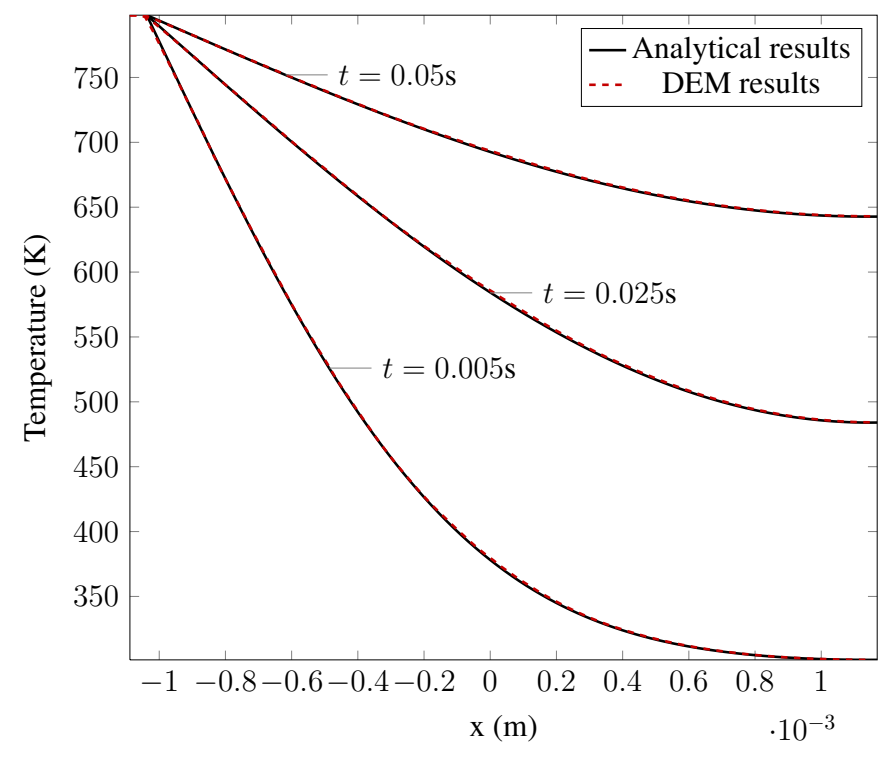

Figure 10: Numerical and analytical temperature distributions at various times in the beam with initial uniform temperature $298 \mathrm{~K}$ and surface temperature $798 \mathrm{~K}$.

$$
\Theta(x, t)=\Theta_{M}-\frac{4\left(\Theta_{M}-\Theta_{m}\right)}{\pi} \sum_{n=0}^{\infty} \frac{(-1)^{n}}{2 n+1} \cos \frac{(2 n+1) \pi x}{2 L} e^{\frac{-\lambda(2 n+1)^{2} \pi^{2} t}{c_{p} \rho 4 L^{2}}}
$$

\subsection{Dynamically heated sheet}

In the second type of simulation, the experiment described below is carried out with both the method developed in this paper and the FEM software Abaqus $6.10^{\mathrm{TM}}$ :

The sheet shown in figure 11, whose properties are described in table 3 , is heated with a moving cylindrical heat source. The 
heat source's characteristics are shown in table 4. The sheet is completely adiabatic. Local temperature evolutions are measured at different check-points $(A, B$ and $C$ ) with both the FEM and DEM. The coordinates of the check-points are given in table 5 .

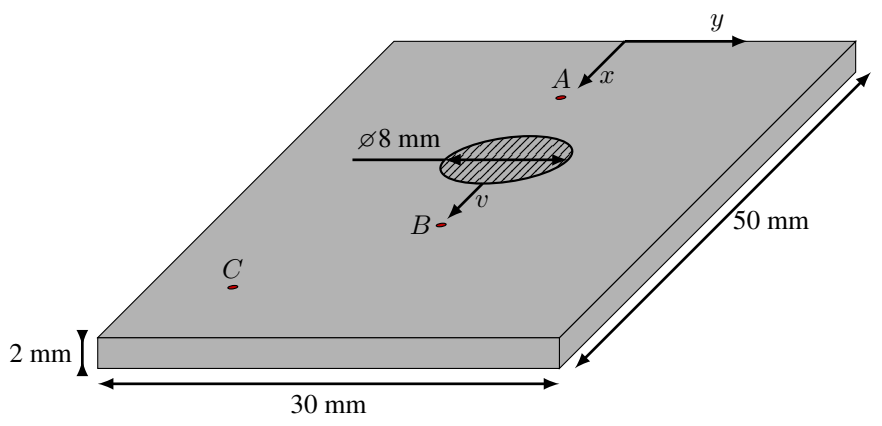

Figure 11: Scheme of the sheet used in calculations.

\begin{tabular}{ll}
\hline Parameter & Value \\
\hline Length & $50 \mathrm{~mm}$ \\
Width & $30 \mathrm{~mm}$ \\
Height & $2 \mathrm{~mm}$ \\
Initial temperature & $25^{\circ} \mathrm{C}$ \\
Density & $2790 \mathrm{~kg} / \mathrm{m}^{3}$ \\
Specific heat & $880 \mathrm{~J} /(\mathrm{kg} \mathrm{K})$ \\
Thermal conductivity & $134 \mathrm{~W} /(\mathrm{m} \mathrm{K})$ \\
\hline
\end{tabular}

Table 3: Sheet's properties.

\begin{tabular}{ll}
\hline Parameter & Value \\
\hline Diameter & $8 \mathrm{~mm}$ \\
Height & $2 \mathrm{~mm}$ \\
Velocity & $3.3 \mathrm{~mm} / \mathrm{s}$ \\
Heat power & $10^{11} \mathrm{~W} / \mathrm{m}^{3}$ \\
Initial $x$ position & $20 \mathrm{~mm}$ \\
\hline
\end{tabular}

Table 4: Heat source's characteristics.

\begin{tabular}{lcc}
\hline Check-point & $x[\mathrm{~mm}]$ & $y[\mathrm{~mm}]$ \\
\hline$A$ & 9.5 & -0.5 \\
$B$ & 31.0 & 0.0 \\
$C$ & 41.5 & -9.5 \\
\hline
\end{tabular}

Table 5: Coordinates of the control points.

The FEM domain is meshed with cubic elements whose edges are $0.5 \mathrm{~mm}$ long. To test the convergence of the DEM calculations, three different type of domains have been created. The first type of domain is composed by 1000 discrete elements with the radius dispersion described in section 4 . The number of discrete elements of the second and third type of domain is 5000 and 20000 respectively. In order to obtain enough data to carry out statistical calculations, five domains of each type have been created and used in the calculations, thus, 15 different discrete domains have been used in total.

For the 5 domains created for a given domain type (1000, 5000 or 20000 discrete elements) we get a different set of temperature evolution at each check-point.

For a given domain type and a given check-point, we can compute the relative dispersion $\epsilon$ of the five sets of temperature evolution:

$$
\epsilon=\frac{1}{N} \sum_{i=1}^{N}\left(\frac{\theta_{i, \max }-\theta_{i, \min }}{\theta_{i, \max }}\right)
$$

where $\theta_{i, \max }=\max _{j=1, \ldots, 5}\left\{\theta_{i, j}\right\}$ and $\theta_{i, \min }=\min _{j=1, \ldots, 5}\left\{\theta_{i, j}\right\}$, being $\theta_{i, j}$ the temperature at time step $i$ in discrete domain $j$ and $N$ the total number of time steps of the simulation. In figure 12 we present the value of $\epsilon$ for the three check-points for each of the three domain types.

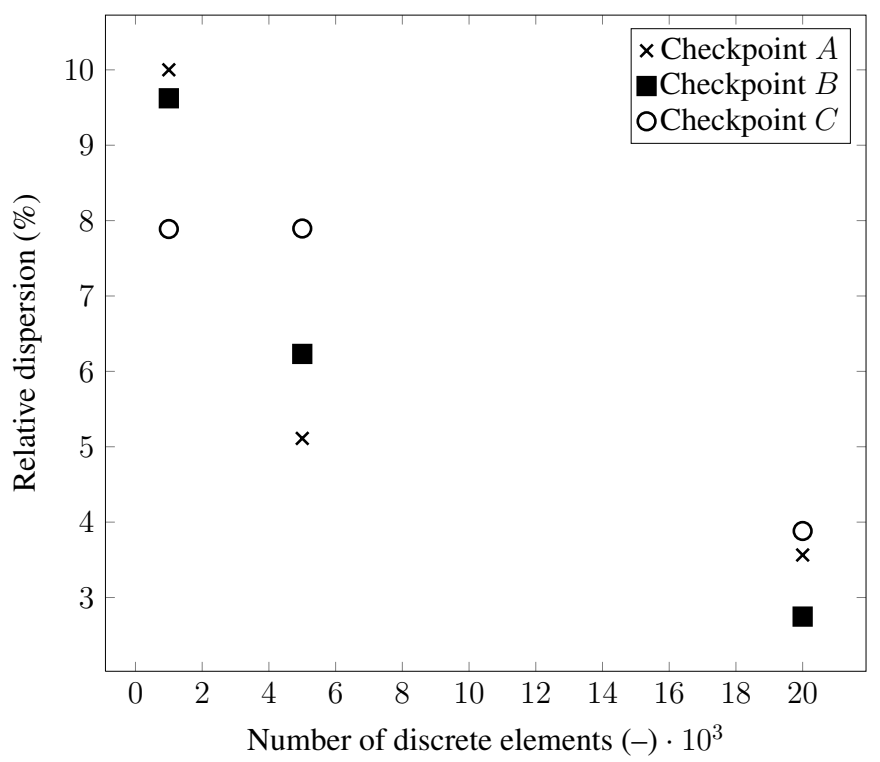

Figure 12: Averaged relative dispersions of results obtained in the check-points for each type of domain.

The dispersion from the five domains created with 20000 discrete elements is considered to be satisfying (less than $4 \%$ ); thus, the average thermal field of the five domains is calculated and compared with the Abaqus $6.10^{\mathrm{TM}}$ results. Figures 13,14 and 15 show this comparison.

The maximal temperature difference is found for every figure, and the relative error between the FEM and DEM results is calculated at that point.

The aim of this simulation is to theoretically validate the method explained in this work using a complex thermal example, including high temperature gradients. Because of this aim, the sheet is allowed to reach elevated temperatures that would be impossible to find experimentally in the simulated material because the sheet's properties are those of an aluminium alloy, which would melt before reaching such high temperatures. 


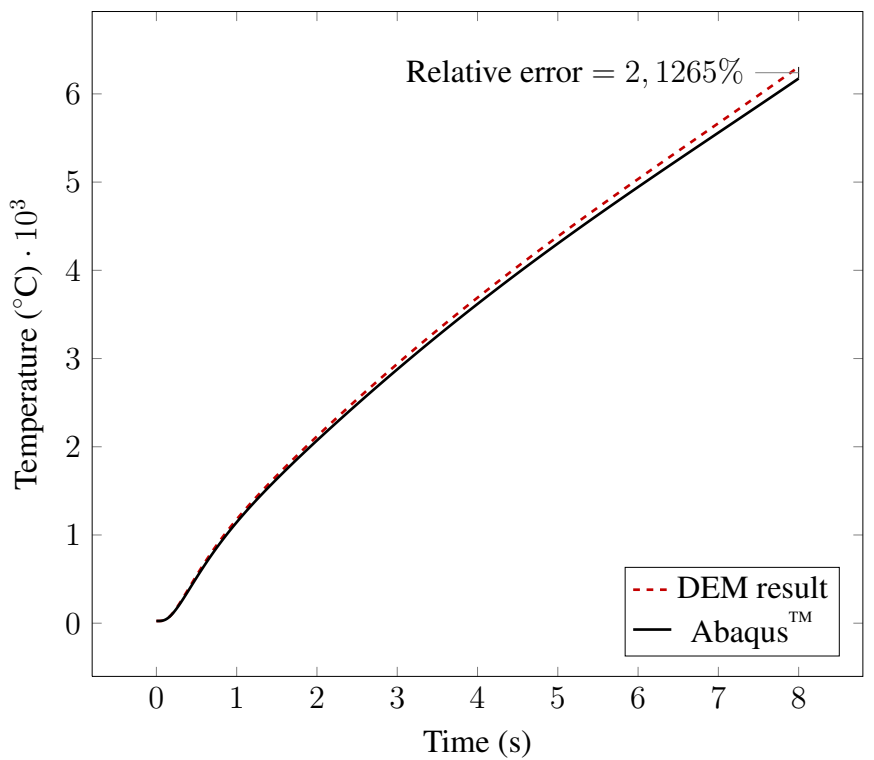

Figure 13: Thermal field obtained at check-point $A$ by the DEM and FEM.

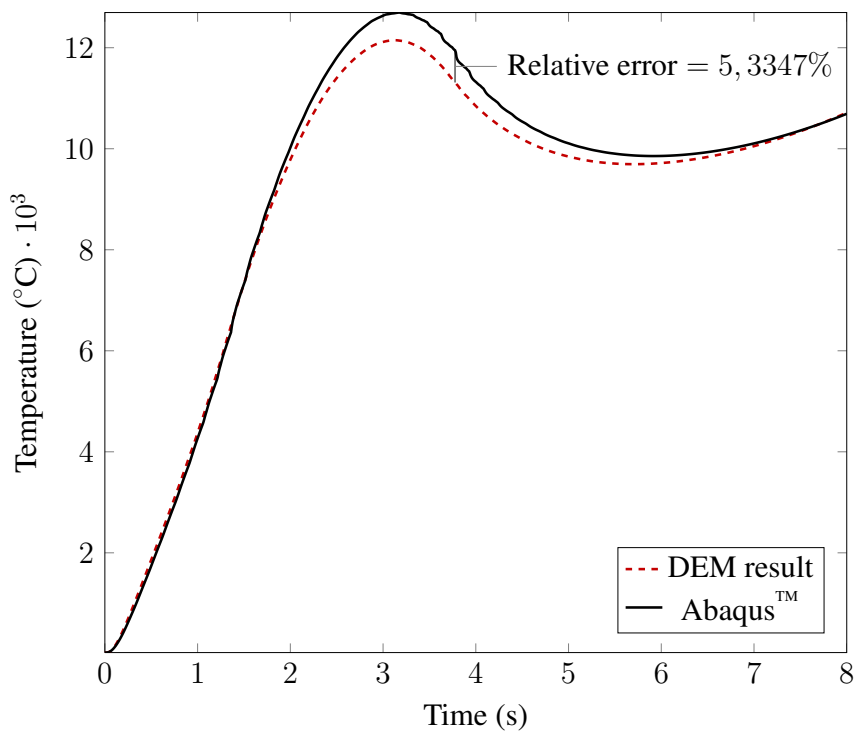

Figure 14: Thermal field obtained at check-point $B$ by the DEM and FEM.

\section{Conclusion \& perspectives}

This work shows the way in which DEM can be used to carry out complex thermal field simulations using 3D, compact, homogeneous, and isotropic (in therms of the contact directions) discrete domains. Those domains can also be used to calculate mechanical fields so that thermo-mechanical simulations are straightforward to implement.

The results show that the relative error between the FEM and DEM results is acceptable, even during a complex thermal simulation involving high temperature gradients.

To reduce the calculation time, a long-term solution will consist of coupling the DEM with continuous approaches such as the FEM or NEM. Once again, the type of domain used in this paper eases this task.

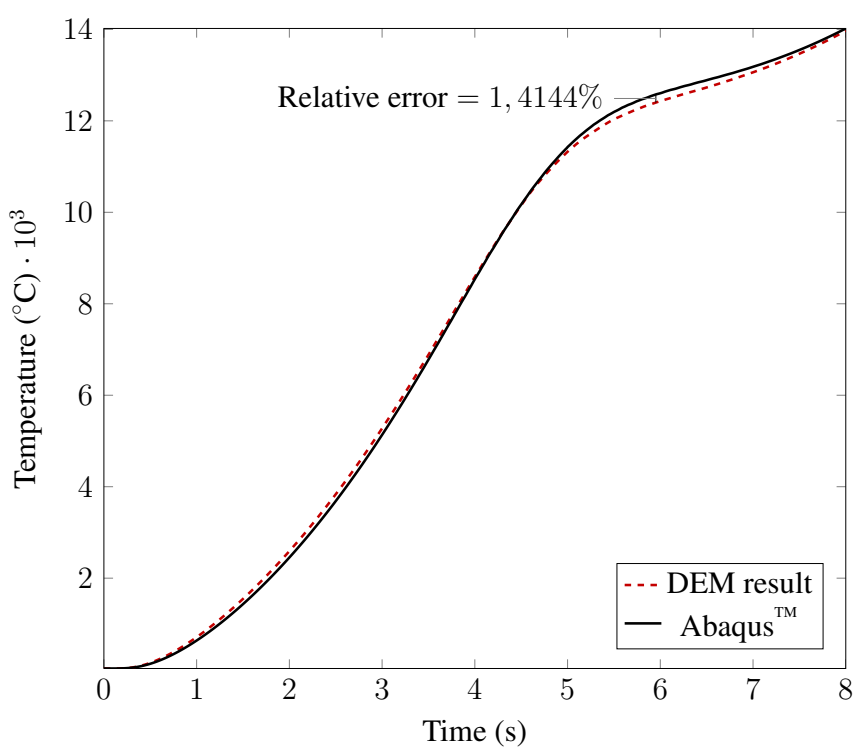

Figure 15: Thermal field obtained at check-point $C$ by the DEM and FEM.

\section{Acknowledgements}

Financial support for this research from the Basque Government (Hezkuntza, Unibertsitate eta Ikerketa sailaren Ikertzaileen Prestakuntzarako Programa) is gratefully appreciated.

\section{References}

[1] J. B. J. FOURIER, Thorie de la chaleur, F. Didot, 1822.

[2] J. CRANK, The Mathematics of Diffusion, 2nd Edition, Oxford University Press, 1956.

[3] O. C. ZIENKIEWICZ, R. L. TAYLOR, J. Z. ZHU, The Finite Element Method: Its Basis and Fundamentals, 6th Edition, ELSEVIER, 2005.

[4] P. A. CUNDALL, A computer model for simulating progressive, largescale movements of blocky rock systems, in: Proceedings of the symposium of the International Society of Rock Mechanics, Vol. 2, 1971.

[5] F. A. TAVAREZ, M. E. PLESHA, Discrete element method for modelling solid and particulate materials, International Journal for Numerical Methods in Engineering 70 (2006) 379-404.

[6] F. FLEISSNER, T. GAUGELE, P. EBERHARD, Application of the discrete element method in mechanical engineering, Multibody System Dynamics 18 (2007) 81-94.

[7] D. RICHARD, I. IORDANOFF, M. RENOUF, Y. BERTHIER, Thermal study of the dry sliding contact with third body presence, ASME Journal of Tribology 130, Issue 3 (031404) (2008) 10.

[8] F. CHINESTA, S. CESCOTTO, E. CUETO, P. LORONG, Natural Element Method for the Simulation of Structures and Processes, ISTE-Wiley, London, 2010.

[9] Z. P. TANG, Three-dimensional DEM theory and its application to impact mechanics, Science in China 44 (6), 2001.

[10] J. E. JONES, On the determination of molecular fields, in: Proceedings of the Royal Society of London, Vol. 106 of A, Containing Papers of a Mathematical and Physical Character, The Royal Society, 1924, pp. 441462.

[11] M. HAHN, T. WALLMERSPERGER, B. H. KRPLIN, Discrete element representation of continua: Proof of concept and determination of the material parameters, Computational Materials Science 50 (2010) 391-402.

[12] D. ANDRÉ, I. IORDANOFF, J. L. CHARLES, J. NAUPORT, Discrete element method to simulate continuous material by using the cohesive beam model, Computer Methods in Applied Mechanics and Engineering 213-216 (2012), pp. 113-125.

[13] W. L. VARGAS, J. J. McCARTHY, Heat conduction in granular materials, AIChE Journal 47 (5) (2001) 1052-1059. 
[14] W. L. VARGAS, J. J. McCARTHY, Stress effects on the conductivity of particulate beds, Chemical Engineering Science 57 (2002) 3119-3131.

[15] W. L. VARGAS, J. J. McCARTHY, Conductivity of granular media with stagnant interstitial fluids via thermal particle dynamics simulation, International Journal of Heat and Mass Transfer 45 (2002) 4847-4856.

[16] W. L. VARGAS, J. C. MURCIA, L. E. PALACIO, D. M. DOMINGUEZ, Fractional diffusion model for force distribution in static granular media, PHYSICAL REVIEW E 68 (021302) (2003) 9.

[17] W. L. VARGAS, J. J. McCARTHY, Thermal expansion effects and heat conduction in granular materials, PHYSICAL REVIEW E 76 (041301) (2007) 8.

[18] M. HAHN, M. SCHWARZ, B. H. KRPLIN, T. WALLMERSPERGER, Discrete element method for the thermal field: Proof of concept and determination of the material parameters, Computational Materials Science 50 (10) (2011) 2771-2784.

[19] T. PÖSCHEL, S. LUDING, Granular gasses, Vol. 564 of Lecture Notes in Physics. 2001. 